\title{
Article
}

\section{Dynamics of Ultracold Bosons in Artificial Gauge Fields-Angular Momentum, Fragmentation, and the Variance of Entropy}

\author{
Axel U. J. Lode ${ }^{1, *(\mathbb{D}, \text { Sunayana Dutta }}{ }^{2,3}$ and Camille Lévêque ${ }^{4,5}$ (i) \\ 1 Physikalisches Institut, Albert-Ludwigs-Universität Freiburg, Hermann-Herder-Straße 3, \\ D-79104 Freiburg, Germany \\ 2 Department of Mathematics, University of Haifa, Haifa 3498838, Israel; sunayanadutta59@gmail.com \\ 3 Haifa Research Center for Theoretical Physics and Astrophysics, University of Haifa, Haifa 3498838, Israel \\ 4 Vienna Center for Quantum Science and Technology, Atominstitut, TU Wien, Stadionallee 2, \\ 1020 Vienna, Austria; camille.leveque@tuwien.ac.at \\ 5 Wolfgang Pauli Institute c/o Faculty of Mathematics, University of Vienna, Oskar-Morgenstern Platz 1, \\ 1090 Vienna, Austria \\ * Correspondence: auj.lode@gmail.com
}

Citation: Lode, A.U.J.; Dutta, S.;

Lévêque, C. Dynamics of Ultracold Bosons in Artificial Gauge Fields-Angular Momentum, Fragmentation, and the Variance of Entropy. Entropy 2021, 23, 392. https://doi.org/10.3390/e23040392

Academic Editor: Jay Lawrence

Received: 15 December 2020

Accepted: 11 March 2021

Published: 25 March 2021

Publisher's Note: MDPI stays neutral with regard to jurisdictional claims in published maps and institutional affiliations.

Copyright: (c) 2021 by the authors. Licensee MDPI, Basel, Switzerland. This article is an open access article distributed under the terms and conditions of the Creative Commons Attribution (CC BY) license (https:// creativecommons.org/licenses/by/ $4.0 /)$.

\begin{abstract}
We consider the dynamics of two-dimensional interacting ultracold bosons triggered by suddenly switching on an artificial gauge field. The system is initialized in the ground state of a harmonic trapping potential. As a function of the strength of the applied artificial gauge field, we analyze the emergent dynamics by monitoring the angular momentum, the fragmentation as well as the entropy and variance of the entropy of absorption or single-shot images. We solve the underlying time-dependent many-boson Schrödinger equation using the multiconfigurational time-dependent Hartree method for indistinguishable particles (MCTDH-X). We find that the artificial gauge field implants angular momentum in the system. Fragmentation-multiple macroscopic eigenvalues of the reduced one-body density matrix - emerges in sync with the dynamics of angular momentum: the bosons in the many-body state develop non-trivial correlations. Fragmentation and angular momentum are experimentally difficult to assess; here, we demonstrate that they can be probed by statistically analyzing the variance of the image entropy of single-shot images that are the standard projective measurement of the state of ultracold atomic systems.
\end{abstract}

Keywords: Boson systems; ultracold gases; trapped gases; dynamic properties of condensates; collective and hydrodynamic excitations; superfluid flow; Bose-Einstein condensates; vortices; topological excitations

\section{Introduction}

Since the first realization of Bose-Einstein condensates in 1995 [1-3], ultracold atoms have become a standard probe for analog quantum simulations-due to the tunability and flexibility of these quantum states of matter, they can be manipulated to behave like other systems, for instance, condensed matter systems which are not as flexible or easy to observe. Popular examples include the realization of the quantum simulation of the superfluid-toMott-insulator transition [4,5], quantized conductance [6,7], the Dicke model [8,9], and magnetism realized via artificial gauge fields for ultracold atoms [10].

Such artificial gauge fields can make the neutral ultracold atoms behave as if they were charged particles experiencing a magnetic field and were investigated experimentally and theoretically with an external lattice potential [11-13] or without one [14-16].

In this paper, we investigate the physics of a two-dimensional system of harmonically trapped interacting ultracold bosons quenched with an artificial magnetic field (AMF) from a many-body point of view. The time-dependent Gross-Pitaevskii mean-field theory $[17,18]$ is the most widespread tool to theoretically model many-body systems of ultracold bosonic 
atoms subject to an AMF. This approach recovers many of the physical phenomena observed, but neglects correlations by its construction using a mean-field ansatz; here, we go beyond mean-field and use the multiconfigurational time-dependent Hartree method for bosons (MCTDH-B) [19-21] to approximate the solution of the Schrödinger equation for ultracold atoms subject to an AMF. MCTDH-B is a method from the MCTDH family of methods [22-27] for indistinguishable particles (MCTDH-X) [22,28-38] that is able to self-consistently describe physics involving the presence and effects of quantum correlations. MCTDH- $X$ was successfully applied to demonstrate the importance of variances of observables [39-43] and of single-shot images $[9,44]$ to the correlations of particles in the many-body state. Using MCTDH-X, intriguing correlation effects beyond the commonly employed Bose-Hubbard description were found to be present in lattices [45-50] and cavitygenerated lattices [51-53] and the breakdown of commonly used mean-field approaches has been demonstrated in tunneling dynamics [54-56] and in harmonic traps [57-59]. A key focus of the applications of MCTDH-B has been the emergence of fragmentation [60-62], where the reduced one-body density matrix has multiple significant eigenvalues, see, for instance, Refs. [54,63-70]. To obtain the results presented in this work, we used the MCTDH-X software hosted at http:/ / ultracold.org (accessed on $\pi$-day, 14 March 2021), see References [38,50,71-74].

Our paper is structured as follows-in Section 2 we introduce the Hamiltonian and the MCTDH-X method we use, in Section 3 we discuss the observables that we are using in Section 4 to investigate the dynamics of ultracold atoms in an AMF; Section 5 summarizes our conclusions and provides an outlook.

\section{Hamiltonian and Methods}

We consider a system of bosonic particles with two-body interactions in two spatial dimensions. The state of the bosons is initialized in the ground state of a parabolic trap without an AMF present. Subsequently, the system is quenched by turning on suddenly an artificial gauge field corresponding to a homogeneous AMF perpendicular to the plane in which the bosons are trapped.

For the sake of clarity of presentation, we will omit the dependence of quantities on time $t$ throughout this work, where it is obvious.

\subsection{Setup}

To setup the time-dependent many-body Schrödinger equation (TDSE), we use the Hamiltonian

$$
\mathcal{H}=\int \mathrm{d} \mathbf{x} \hat{\Psi}^{\dagger}(\mathbf{x})[T(\mathbf{x})+V(\mathbf{x})] \hat{\Psi}(\mathbf{x})+\frac{1}{2} \int \mathrm{d} \mathbf{x} \mathrm{d} \mathbf{x}^{\prime} \hat{\Psi}^{\dagger}(\mathbf{x}) \hat{\Psi}^{\dagger}\left(\mathbf{x}^{\prime}\right) W\left(\mathbf{x}, \mathbf{x}^{\prime}\right) \Psi\left(\mathbf{x}^{\prime}\right) \hat{\Psi}(\mathbf{x}) .
$$

Here, we work in atomic units ( $\hbar=m=1$ ), the potential $V(\mathbf{x})$ [with $\mathbf{x}=(x, y)$ ] is chosen to be harmonic, $V(\mathbf{x})=\frac{1}{2} \mathbf{x}^{2}$, and we consider contact interactions $W\left(\mathbf{x}, \mathbf{x}^{\prime}\right)=g_{0} \delta\left(\mathbf{x}-\mathbf{x}^{\prime}\right)$. Formally, one cannot use a contact interaction in two spatial dimensions with a complete basis set since the outcome would be that of the noninteracting bosons $[75,76]$. In simple terms, this is because the integral measure of the support of the Dirac- $\delta$ is zero for two and more spatial dimensions. For a proof, the interested reader is deferred to Ref. [76] and for an example for finding non-zero ranged Gaussian interaction potentials with similar physical behavior for ultracold bosons, see Ref. [75].

In the present work, we employ a finite truncation of the many-body basis (chiefly $M=4$ orbitals) and aim to demonstrate that beyond-mean-field phenomena do emerge. The kinetic energy is augmented with an artificial gauge field $\mathbf{A}(\mathbf{x} ; t)$ :

$$
\hat{T}(\mathbf{x})=\frac{1}{2}\left(-i \nabla_{\mathbf{x}}-g \mathbf{A}(\mathbf{x} ; t)\right)^{2} .
$$


For simplicity, we consider the case of unit charge $g=1$ and a homogeneous magnetic field $\mathbf{B}$ in $z$-direction of strength $B(\mathbf{x} ; t)$ :

$$
\mathbf{B}(\mathbf{x} ; t)=B(t) \hat{e}_{z} .
$$

Here, $\hat{e}_{z}$ denotes the unit vector in $z$-direction. In the following, we work in Landau gauge,

$$
\mathbf{A}(\mathbf{x} ; t)=B(t) \hat{e}_{x},
$$

and consider a quench scenario in the following, that is,

$$
B(t)=B \Theta(t) .
$$

Here, $\Theta(t)$ denotes the Heaviside step function, that is, the magnetic field is suddenly turned on at $t>0$ after the system has been initialized, see Figure 1 for a sketch.

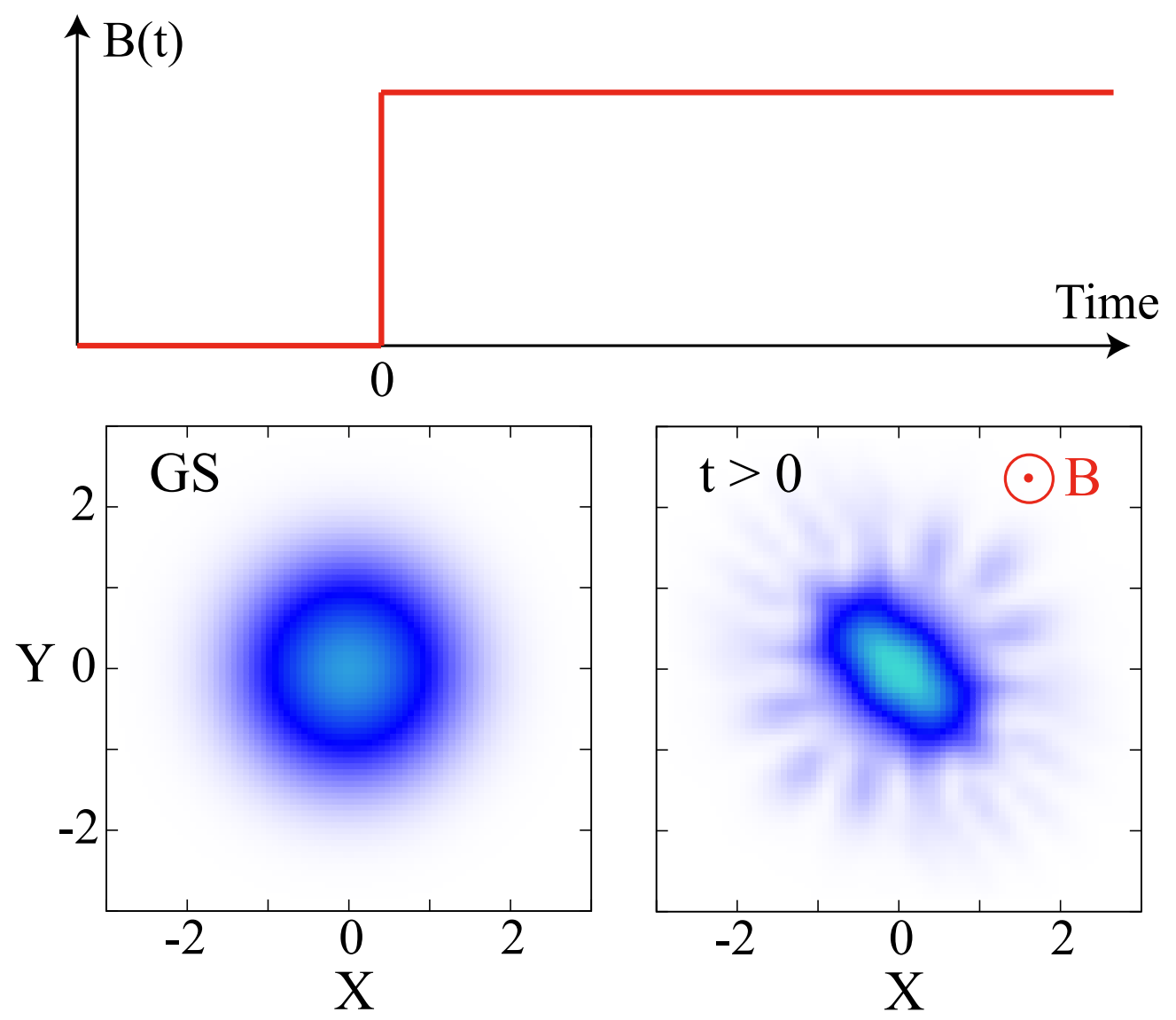

Figure 1. Sketch of our setup. Two-dimensional ultracold bosonic particles are prepared in the ground state of an isotropic harmonic trap (label "GS") for time $t \leq 0$. At $t>0$, an artificial magnetic field pointing in the Z-direction perpendicular to the ultracold atoms of strength $B(t)$ (top) is switched on suddenly. This quench triggers many-body dynamics of the state (label " $t>0$ ").

In our investigation, we analyze the many-body dynamics by monitoring observables as a function of the effective magnetic field strength $B$ at $t>0$ after the quench.

\subsection{Method}

To solve the TDSE,

$$
\mathcal{H}|\Psi\rangle=i \partial_{t}|\Psi\rangle,
$$


we use the multiconfigurational time-dependent Hartree method for indistinguishable particles [19-21,38,50,71-73]. Regarding our notation in Equation (1), the MCTDH-X method implies that the field operators are represented by a sum of $M$ time-dependent singleparticle states or orbitals:

$$
\hat{\Psi}(\mathbf{x})=\sum_{j=1}^{M} \hat{b}_{j} \phi_{j}(\mathbf{x} ; t) .
$$

This corresponds to the following ansatz for the wavefunction:

$$
|\Psi\rangle=\sum_{\vec{n}} C_{\vec{n}}|\vec{n} ; t\rangle .
$$

For bosons, the summation runs on all

$$
\left(\begin{array}{c}
N+M-1 \\
N
\end{array}\right)
$$

symmetric time-dependent configurations $\vec{n}=\left(n_{1}, \ldots, n_{M}\right)$ with fixed particle number $N=\sum_{i=1}^{M} n_{i}$. To derive the MCTDH-X equations, the ansatz in Equation (8) is plugged in a suitable variational principle [31,77-81]. The resulting equations are a two coupled sets-A set of linear equations for the coefficients $\left\{C_{\vec{n}}(t)\right\}$ and a set of non-linear ones for the orbitals $\left\{\phi_{j}(\mathbf{x} ; t) ; j=1, \ldots, M\right\}$, see Appendix A for the equations and References [19-21,38,71-73] for details and derivation.

\section{Quantities of Interest}

Here, we define the observables that we use to quantify the dynamics of $N$-boson systems: the one-body density, the eigenvalues of the reduced one-body density matrix $(1 \mathrm{BDM})$, the angular momentum, and the image entropy and its variance evaluated from simulated single-shot images.

\section{Density, One-body density matrix, and Natural Occupations:}

The $1 \mathrm{BDM}$ is a hermitian matrix defined as

$$
\rho^{(1)}\left(\mathbf{x}, \mathbf{x}^{\prime}\right)=\left\langle\Psi\left|\hat{\Psi}(\mathbf{x}) \hat{\Psi}^{\dagger}\left(\mathbf{x}^{\prime}\right)\right| \Psi\right\rangle=\sum_{k, q} \rho_{k q} \phi_{k}^{*}(\mathbf{x} ; t) \phi_{q}(\mathbf{x} ; t) .
$$

Here, we used the matrix elements $\rho_{k q}=\left\langle\Psi\left|\hat{b}_{k}^{+} \hat{b}_{q}\right| \Psi\right\rangle$ to represent the 1BDM using the orbitals corresponding to the creation and annihilation operators $\hat{b}_{k}^{+}$and $\hat{b}_{q}$, respectively. The diagonal of the one-body density matrix is referred to as the density $\rho(\mathbf{x})$ :

$$
\rho(\mathbf{x})=\rho^{(1)}\left(\mathbf{x}, \mathbf{x}^{\prime}=\mathbf{x}\right) .
$$

The density $\rho(\mathbf{x})$ is a real quantity and has no phase, because it is the diagonal of a hermitian matrix, $\rho^{(1)}\left(\mathbf{x}, \mathbf{x}^{\prime}\right)$. The eigenvalues of the 1BDM, Equation (9), can be obtained via a diagonalization that corresponds to a unitary transformation of the orbitals $\phi_{j}(\mathbf{x} ; t)$ to the so-called natural orbitals $\phi_{j}^{(N O)}(\mathbf{x} ; t)$ :

$$
\frac{\rho^{(1)}\left(\mathbf{x}, \mathbf{x}^{\prime}\right)}{N}=\sum_{j} \lambda_{j} \phi_{j}^{(N O), *}(\mathbf{x} ; t) \phi_{j}^{(N O)}\left(\mathbf{x}^{\prime} ; t\right) .
$$

The eigenvalues $\lambda_{j}$ are normalized, $\sum_{j=1}^{M} \lambda_{j}=1$ and, without loss of generality, we consider them to be sorted by size, $\lambda_{1} \geq \lambda_{2} \geq \ldots$, throughout this work. The eigenvalues $\lambda_{j}$ (natural occupations) determine the degree of condensation and fragmentation of the system. Bosons with a 1BDM with only a single contributing eigenvalue $\lambda_{1}$ are condensed [82] and 
bosons with a $1 \mathrm{BDM}$ with multiple macroscopic eigenvalues contributing $\lambda_{1} \sim \mathcal{O}(N) ; \lambda_{2} \sim$ $\mathcal{O}(N) ; \ldots$ are fragmented $[60,61]$.

The eigenvalues of the 1BDM can be used as a precursor of correlations in the state $|\Psi\rangle$. This can be seen using, for instance, the Glauber first-order correlation function [83],

$$
\left|g^{(1)}\left(\mathbf{x}, \mathbf{x}^{\prime}\right)\right|=\left|\frac{\rho^{(1)}\left(\mathbf{x}, \mathbf{x}^{\prime}\right)}{\sqrt{\rho(\mathbf{x}) \rho\left(\mathbf{x}^{\prime}\right)}}\right| .
$$

If the system is in a condensed state, the 1BDM has only a single eigenvalue and is therefore a product of a single (complex-valued) orbital, $\rho^{(1)}\left(\mathbf{x}, \mathbf{x}^{\prime}\right) \propto \phi_{1}^{*}(\mathbf{x}) \phi_{1}\left(\mathbf{x}^{\prime}\right)$. It follows that $\left|g^{(1)}\left(\mathbf{x}, \mathbf{x}^{\prime}\right)\right|$ is constant for all $\left(\mathbf{x}, \mathbf{x}^{\prime}\right)$-for particles in a condensed state correlations are absent. It is instrumental to note here, that this condensed, single-orbital case with absent correlations is presupposed in mean-field approaches like the time-dependent GrossPitaevskii theory $[17,18]$. Similarly, when $\rho^{(1)}\left(\mathbf{x}, \mathbf{x}^{\prime}\right)$ is a sum of two or more orbitals as in Equation (9) then it can no longer be represented simply using its diagonal, the density $\rho^{(1)}\left(\mathbf{x}, \mathbf{x}^{\prime}=\mathbf{x}\right)=\rho(\mathbf{x})$. Furthermore, in this fragmented case, the denominator of Equation (12) is a product of two weighted sums of orbitals, $\rho(\mathbf{x})$, and $\rho\left(\mathbf{x}^{\prime}\right)$, respectively. This product involves non-trivial cross terms and the correlation function attains a value $\left|g^{(1)}\left(\mathbf{x}, \mathbf{x}^{\prime}\right)\right| \leq 1$ for all $\left(\mathbf{x}, \mathbf{x}^{\prime}\right)$-for particles in a fragmented state, correlations are present and the single-orbital picture of mean-field approaches like the time-dependent GrossPitaevskii theory $[17,18]$ cannot be applied.

\section{Angular momentum:}

The angular momentum operator in $\hat{e}_{z}$-direction for a two-dimensional system is defined as

$$
\hat{L}_{z}=\hat{e}_{z} \cdot(\hat{x} \times \hat{p})=-i\left(\hat{x} \hat{\partial}_{y}-\hat{y} \hat{\partial}_{x}\right) .
$$

Bosonic quantum systems with angular momentum are rich in physics: they feature condensed vortices $[17,18,84]$, phantom vortices $[69,85]$, spatially partitioned many-body vortices [68,86], and fragmentation [68,69,85-88]. Since phantom vortices are the most pronounced characteristic feature of angular momentum which we find in our study below, we discuss them in the following. Typically, the term "vortex" refers to a topological defect in the density of quantum system connected with a discontinuity in the phase. A phantom vortex is an analog of such a conventional vortex, but for a natural orbital. A phantom vortex thus represents a topological defect connected with a discontinuity in the phase in one of the field modes of a many-particle states that corresponds to a natural orbital. Phantom vortices were shown to emerge as topological defects in the correlation function in Reference [69]. Moreover, in the common detection scheme for cold atoms, single-shot images (see below) they show as topological defects whose position fluctuates from image to image, see Reference [85].

\section{Single shots, image entropy and its variance:}

To assess the observability of the emergent physics in experimental setups with ultracold atoms, we simulate the detection of our numerical model wavefunctions in absorption or single-shot images $[9,85,89]$. A set of $N_{s}$ single shots,

$$
\mathbf{S}^{j}=\left(\mathbf{s}_{1}^{j}, \ldots, \mathbf{s}_{N}^{j}\right) ; \quad j=1, \ldots, N_{s},
$$

is nothing but $N_{s}$ random samples that are $N$-variate and distributed according to the $N$-particle probability given by $|\Psi|^{2}$,

$$
P\left(\mathbf{x}_{1}, \ldots, \mathbf{x}_{N}\right)=\left|\Psi\left(\mathbf{x}_{1}, \ldots, \mathbf{x}_{N}\right)\right|^{2} .
$$

To generate images from these single shots, we convolute them with a point spread function. Typical choices include Gaussian (see $[9,44,49,85]$ ) or even quantum point spread 
functions [90]. Here, for simplicity, we consider the idealized case of a $\delta$-shaped point spread function to obtain our single-shot images:

$$
\mathcal{S}^{j}(\mathbf{x})=\sum_{i=1}^{N} \delta\left(\mathbf{x}-\mathbf{x}_{i}^{j}\right)
$$

We will consider the image entropy $\zeta$ of single-shot images of the state $|\Psi\rangle$ :

$$
\zeta=-\frac{1}{N_{s}} \sum_{j=1}^{N_{s}} \zeta^{j} ; \quad \zeta^{j}=\int d \mathbf{x} \mathcal{S}^{j}(\mathbf{x}) \ln \mathcal{S}^{j}(\mathbf{x}) .
$$

In the limit of large $N_{s}$, the image entropy $\zeta$ is equivalent to the density-entropy studied, for instance, in Reference [91]. Fundamentally, the image entropy is a measure for the information content in the particle distributions detected in single-shot images. While Ref. [91] found the entropy to be connected to the presence of correlations in the state, we found it not to be a conclusive pointer in our present work. The variances of observables, however, serve as a precursor of quantum fluctuations and correlations in many-body systems [39-43]; we are thus motivated to also analyze the variance $\sigma_{\zeta}$ of the image entropy $\zeta$ :

$$
\sigma_{\zeta}=\frac{1}{N_{s}} \sum_{j=1}^{N_{s}}\left[\zeta-\zeta^{j}\right]^{2}
$$

\section{Results}

We now carve out the connection between artificial gauge fields and many-body correlations. For this purpose we focus on the dynamics of a model system of $N=$ 100 two-dimensional ultracold bosonic atoms with an interaction strength of $g_{0}=0.05$ [cf. Equations (1)-(5) for $t \leq 0$ ]. We now make an example for realizing this interaction $g_{0}$ with a real trap configuration with ${ }^{87} \mathrm{Rb}$ in analogy to the study [69] - the Hamiltonian in Equation (1) is multiplied by $\frac{\hbar}{m L^{2}}$, where $m=1.44 \times 10^{-25} \mathrm{~kg}$ is the mass of a ${ }^{87} \mathrm{Rb}$ and $L$ is a length scale that we choose to be $L=0.75 \times 10^{-6} \mathrm{~m}$. This sets our trapping frequency to $\omega=(2 \pi) 207 \mathrm{~Hz}$ and yields a unit of time, $\frac{m L^{2}}{\hbar}$ of $4.84 \mathrm{~ms}$. The total interval of time we consider in the following $t \in[0,200]$ thus corresponds to $0.97 \mathrm{~s}$. In quasi-twodimensional setups, the interaction parameter $g_{0}=2 \sqrt{2 \pi} \frac{a_{s}}{l_{z}}$ depends on the transversal oscillator length, $l_{z}=\sqrt{\frac{\hbar}{m \omega_{z}}}$, and the scattering length of ${ }^{87} \mathrm{Rb}, a_{s}=90.4 a_{0} ;$ here, $\omega_{z}$ is the transversal trapping frequency and $a_{0}$ the Bohr radius. The two-dimensional interaction strength we use, $g_{0}=0.05$, is obtained for a transversal trapping of $\omega_{z} \approx(2 \pi) 3.178 \mathrm{kHz}$. We remark here, that our choice of $g_{0}$ corresponds to a weak interaction; the healing length $\xi=\frac{1}{2 g_{0}(N-1)} \approx 0.101$ is comparable to the oscillator length, that is, 1 in our units.

The system is initialized in its ground state and its dynamics $(t>0)$ are then triggered by suddenly turning on an AMF of strength $B$ [Equation (5)]. In what follows, we aim at an understanding of how the strength of the AMF affects the emergent dynamical behavior. For this purpose, in the main text, we solved the time-dependent many-body Schrödinger equation with MCTDH-X using $M=4$ orbitals [176,581 configurations in the state in Equation (8)] and $128 \times 128$ DVR functions ("modes") to represent each of the orbitals $\left\{\phi_{j}(\mathbf{x} ; t)\right\}$; the total number of optimized and fully time-dependent parameters is thus $176581+4 \times 128^{2} \times 4=242117$. For a convergence study with different $M$ and $N$ see Appendix B.

We open the exposition of our findings with the density $\rho(\mathbf{x})$ and its decomposition into natural orbitals $\phi_{j}^{(\mathrm{NO})}$.

Besides a slight deformation of the density and the orbitals, little effects are seen in Figure $2 \mathrm{a}-\mathrm{e}$ for a weak AMF, $B=1.0$. The phases $\beta_{1 / 2}(\mathbf{x})$, Figure $2 \mathrm{f}-\mathrm{g}$, hint there are no 
phantom vortices. The phases $\beta_{3 / 4}(\mathbf{x})$ feature topological defects aligned with zeros in $\phi_{3 / 4}(\mathbf{x})$, but these orbitals are occupied only by 0.1 particles.

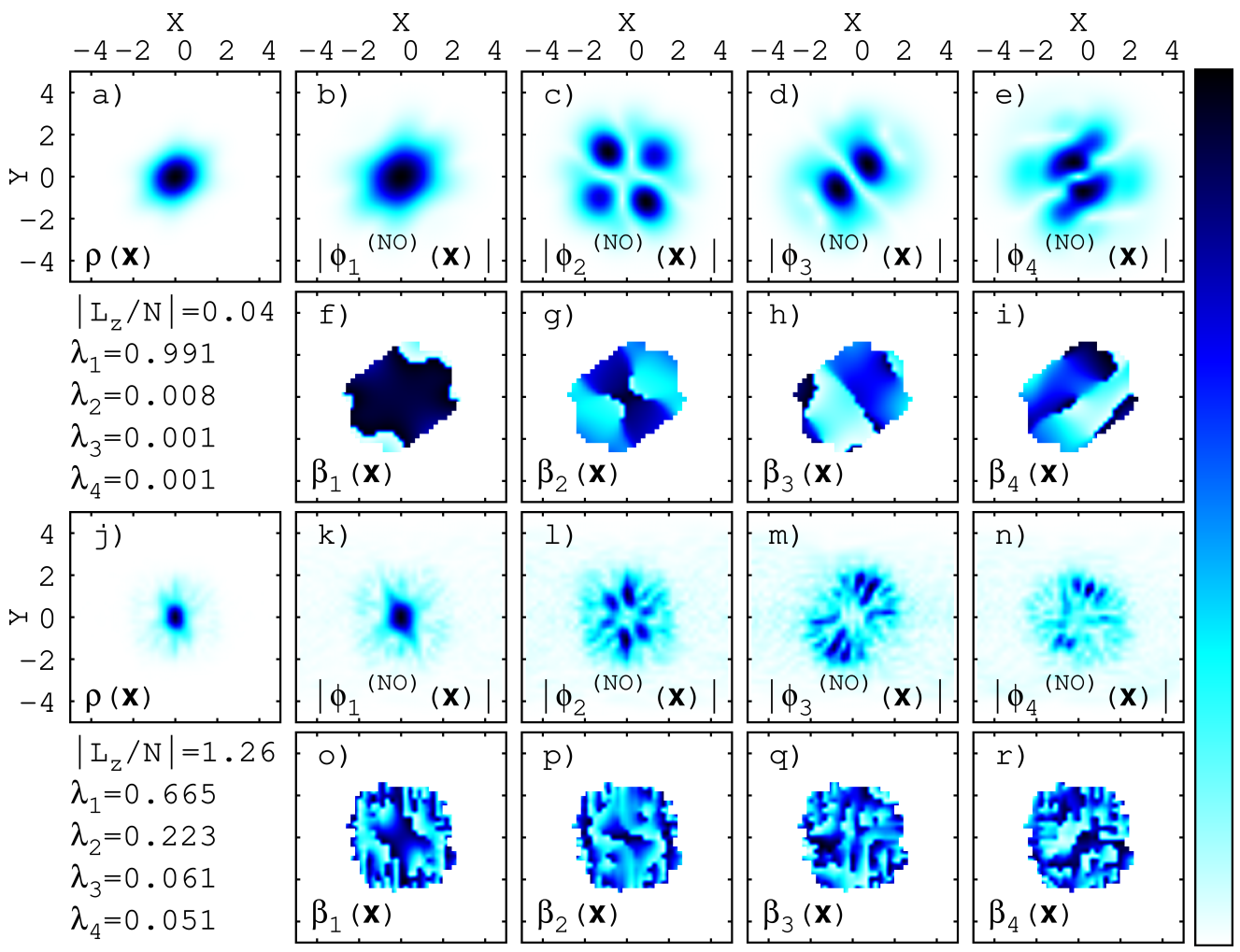

Figure 2. One-body density, natural orbitals, and natural orbital phases for two distinct AMF strengths: $B=1.0$ (weak) in $(\mathbf{a}-\mathbf{i})$ and $B=6.5$ (strong) in $(\mathbf{j}-\mathbf{r})$. The figure shows the quantities at time $t=50.0$. For guidance, the angular momentum per particle and the natural occupations are listed below (a) and (j) for weak and strong AMFs, respectively. The phase plots $\left[\beta_{j}(\mathbf{x})\right.$ in $(\mathbf{f}-\mathbf{i})$ and $(\mathbf{o}-\mathbf{r})]$ are restricted to areas where $\rho(\mathbf{x})>0.01$. The plot ranges are $[0, \sim 0.2]$ for $(\mathbf{a})$ and $(\mathbf{j}),[0, \sim 0.1]$ for $(\mathbf{b}-\mathbf{e})$ and $(\mathbf{k}-\mathbf{n})$, and $[-\pi, \pi]$ for $(\mathbf{o}-\mathbf{r})$. See text for further discussion.

For a comparatively strong AMF, $B=6.5$, in contrast, vortices at the edges of the density (so-called "ghost vortices" [92]) and phantom vortices [69] in the orbitals emerge in Figure $2 \mathrm{j}-\mathrm{r}$-zeros of the orbital densities are accompanied by topological defects in their phase [compare Figure 2, panels (k) and (o), (l) and (p), (m) and (q), (n) and (r), respectively].

These features of the density, orbitals, and their phases are hallmarks of the angular momentum that is deposited in time by the action of a sufficiently strong AMF-for increasing AMF strength $B$, the expectation value $L_{z}=\left\langle\Psi\left|\hat{L}_{z}\right| \Psi\right\rangle$ of the angular momentum operator $\hat{L}_{z}$ [Equation (13)] increases. For instance, we find $L_{z} / N=0.04$ at time $t=50.0$ and $B=1.0$ in Figure $2 \mathrm{a}-\mathrm{i}$ and $L_{z} / N=1.26$ at time $t=50.0$ for $B=6.5$ in Figure $2 \mathrm{j}-\mathrm{r}$ ].

To quantify the dynamics of angular momentum triggered by quenches of the AMF a bit better, we plot $L_{z} / N$ for our system as a function of evolution time and as a function of the strength of the AMF in Figure 3.

We find from Figure $3 a$ that a threshold AMF strength of about $B \gtrsim 6$ is required to generate states with significant angular momentum at long evolution times (here, $t=200$ ). Furthermore, the average angular momentum content increases as the strength of the AMF does. We remark here that the angular momentum features an oscillatory behavior for all AMF strengths that we investigated. This can be understood as a consequence of our quench scenario- the initial state is an eigenstate of the Hamiltonian in Equation (1) for time $t<0$ and has vanishing angular momentum $L_{z}=0$. At $t=0$, the Hamiltonian and its spectrum is changed abruptly due to presence of the AMF, Equation (5). The dynamics of the initial state is thus dependent on these changes in the spectrum of the Hamiltonian by the AMF. At low AMF $(B \lesssim 6)$ strength, the time-evolution of $\left|L_{z}\right|$ quasi-periodically 
goes back to $L_{z}=0$ and is dominated by oscillations at a single frequency [Figure 3b]; we infer that only very few states with $\left|L_{z}\right| \neq 0$ are contributing. The dynamics of the state $|\Psi\rangle$ of the many-boson system is thus a superposition of a very small number of eigenstates of the Hamiltonian after the quench in this quasi-adiabatic case.

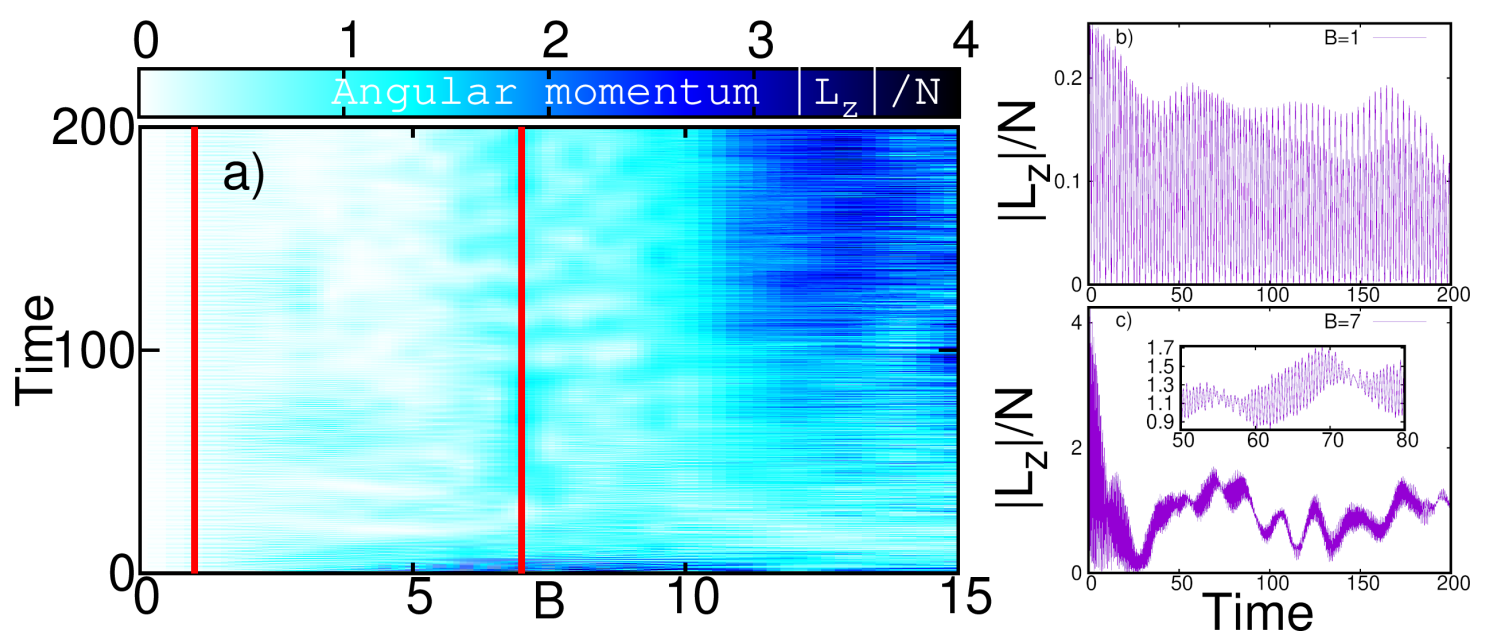

Figure 3. Angular momentum as a function of propagation time and strength of artificial magnetic field (AMF). The expectation value $L_{z} / N=\left\langle\Psi(t)\left|\hat{L}_{z}\right| \Psi(t)\right\rangle / N$ [cf. Equation (13)] is shown as a function of time $t$ and AMF strength $B$ in (a). Interestingly, there is a drastic short-term increase following a subsequent equilibration of $L_{z} / N$ for $5 \lesssim B \lesssim 12$. To highlight the oscillatory nature of the angular momentum we plot the cuts at $B=1$ and $B=7$ [highlighted by the red vertical lines in a)] in panels $(\mathbf{b}, \mathbf{c})$, respectively. The angular momentum returns to $L_{z}=0$ quasi-periodically for $B \lesssim 6$ [as in (b)], but not for $B \gtrsim 6$ [as in (c)]. See text for discussion.

The situation changes for $B \gtrsim 6$, where the time-evolution of $\left|L_{z}\right|$ does not return to $L_{z}=0$ quasi-periodically. In this case, the time-evolution of $\left|L_{z}\right|$ still has its shortest-time oscillations of roughly the same amplitude as for the quasi-adiabatic case $B \lesssim 6$ [Figure 3c]. However, several large-amplitude oscillations with other frequencies contribute. We infer, that the dynamics of the state $|\Psi\rangle$ of the many-boson system is thus a superposition of a large number of states of the Hamiltonian after the quench, in this genuinely non-adiabatic evolution for $B \gtrsim 6$.

We remark here that our results on the oscillatory behavior of the angular momentum in time render it impossible to approach the physics of the many-body state using a co-rotating frame at a certain angular frequency as done in References [62,93].

In References [62,68,69,85,87,88,93-97], an intricate connection of angular momentum content and the presence of correlations or the fragmentation of many-boson states has been pointed out. This motivates us to analyze the time-evolution of the eigenvalues of the reduced one-body density matrix as a precursor of correlations and the departure of the analyzed state from a mean-field description; we, thus, underpin the limitations of a mean-field description, see Figure 4 for a plot of $\lambda_{j}$ as a function of time and strength $B$ of the AMF.

Our present findings for a quenched AMF are in line with results obtained with a time-dependent and slow transfer of angular momentum via a rotating asymmetry of the harmonic trapping potential $[69,85]$ : the dynamical departure from a single-eigenvalue $1 \mathrm{BDM}$ to a fragmented many-body state with correlations accompanies the dynamical acquisition of a significant amount angular momentum. In particular, the region in time and AMF strength, with a quasi-adiabatic evolution of small $\left|L_{z}\right|$ in Figure 3 agrees roughly with the regions with small fragmentation in Figure 4. Conversely, the region in time and AMF strength, with a non-adiabatic evolution of large $\left|L_{z}\right|$ in Figure 3 agrees roughly with the regions with substantial fragmentation in Figure 4. 


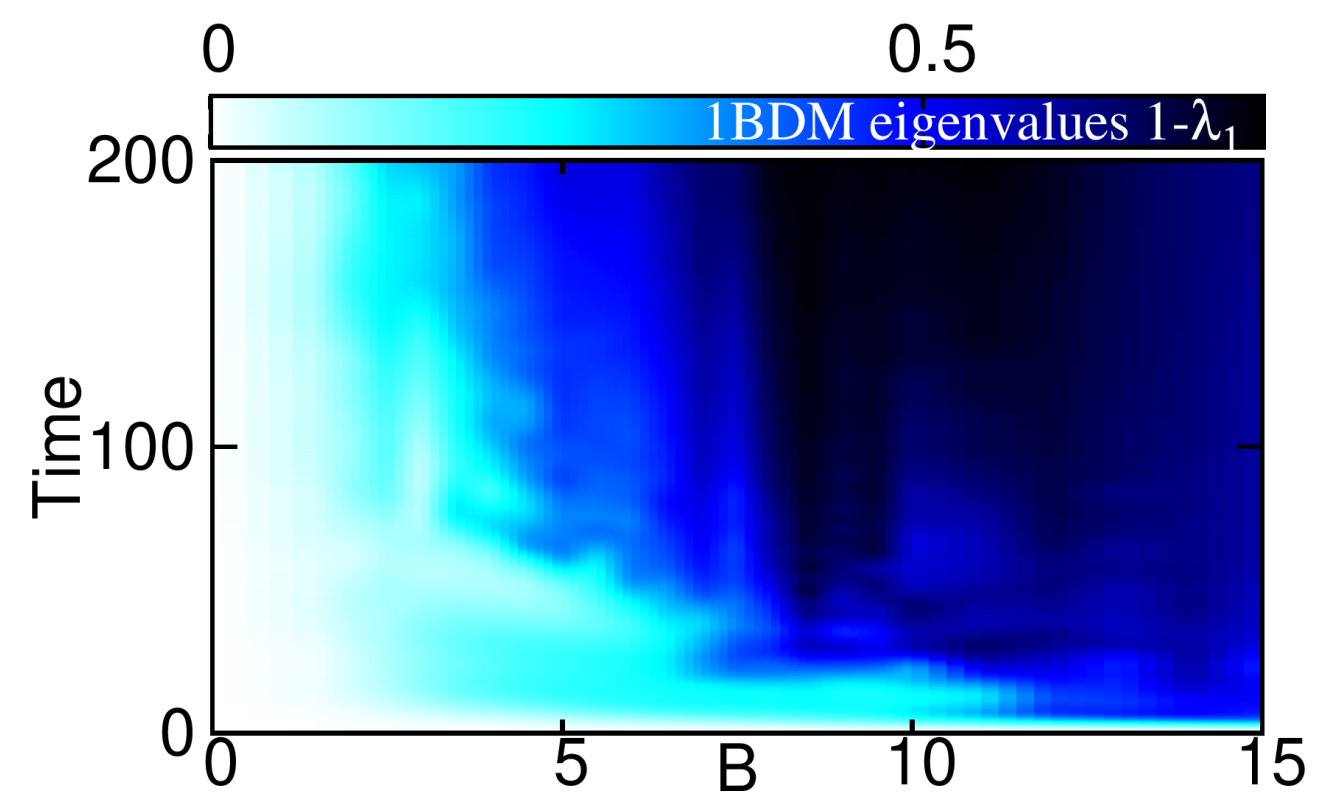

Figure 4. Eigenvalues of the reduced one-body density matrix. The dynamical emergence of multiple significant eigenvalues, i.e., the fragmentation of the many-body state, is quantified here via the time-dependent depletion $1-\lambda_{1}=\sum_{k=2}^{M} \lambda_{k}$ and is in sync with the dynamics of angular momentum (cf. Figure 3).

We now turn to the question of the possibility of an experimental detection of the emergent behavior of angular momentum and the eigenvalues of the one-body density matrix. For this purpose we simulated $N_{s}=1000$ single-shot images for all the many-body wavefunctions $|\Psi(t)\rangle$ for every time in $t \equiv k \cdot d t \in[0,200]$ in steps of $d t=1.0$. From this dataset of single-shot images, we computed the image entropy and its variance $\sigma_{\zeta}$ [Equation (18)]; see Figure 5.

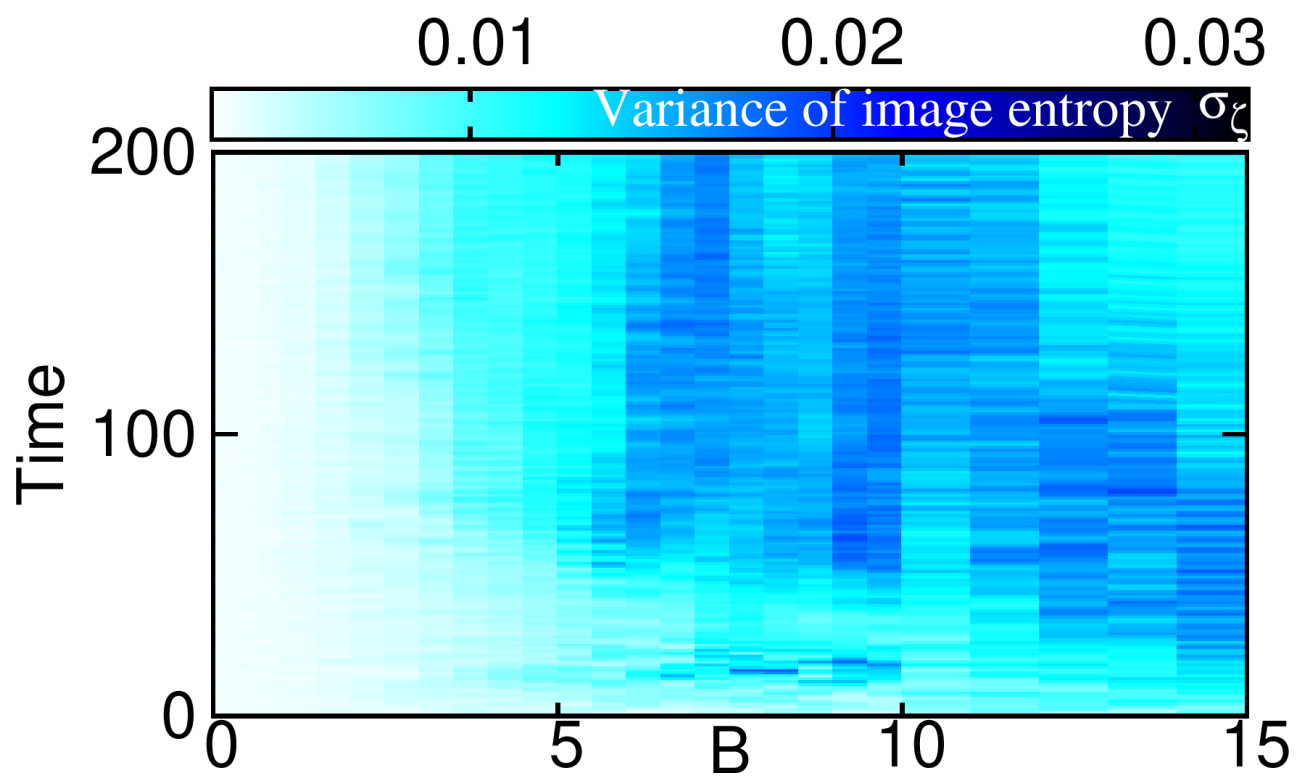

Figure 5. Variance of the image entropy as a function of propagation time and strength of the AMF. The variance of image entropy $\sigma_{\zeta}$ tracks the behavior of the natural occupations and the angular momentum in, respectively, Figures 3 and 4 closely, see text for discussion.

The variance $\sigma_{\zeta}$ becomes significant for the same values of time and AMF strength where the natural occupations in Figure 4 herald the emergence of fragmentation and correlations. We emphasize here, that fragmentation implies the inapplicability of mean- 
field descriptions that are restricted to fully condensed and uncorrelated states, as already described in Section 3. By itself it is an interesting result that the variance of image entropy represents an experimentally feasible way to study the emergence of fragmentation in ultracold bosonic systems. Moreover, we observe that the region in time and AMF strength $B$, where $\left|L_{z}\right|$ features quasi-adiabatic (non-adiabatic) dynamics in Figure 3, coincides roughly with the region where fragmentation and image entropy are low (large) in Figures 4 and 5, respectively. We therefore conjecture that an experimental detection of the presence of a non-adiabatic evolution of angular momentum is feasible by measuring the variance of the image entropy.

We remark that we have not shown complementary results on the image entropy $\zeta$ [Equation (17)] as a function of AMF strength and time, because it shows only very little overall variation. We noted that the entropy $\zeta$ is lowest, where its variance (Figure 5 ) is changing the most. The exploration of a possible fundamental connection of the gradient of the image entropy and the variance $\sigma_{\zeta}$ goes beyond the exploratory scope of our present investigation.

\section{Conclusions and Outlook}

We analyzed the dynamics of interacting two-dimensional ultracold bosonic particles triggered by a quench of an artificial gauge field. We used the multiconfigurational timedependent Hartree method for indistinguishable particles software (http:/ / ultracold.org) (accessed on $\pi$-day, 14 March 2021) to solve the many-body Schrödinger equation from first principles. Our exploratory investigation demonstrates that fragmentation emerges due to the quench if the artificial magnetic field is sufficiently strong. Such an emergence of fragmentation entails the breakdown of conventionally-used mean-field descriptions and, therewith, the occurrence of many-body correlations. We underpin our results by checking their consistency across different quality levels of our MCTDH-X approximation and different numbers of particles.

We have portrayed how correlations show in the expectation value of the angular momentum operator and in the orbitals and their phases as phantom vortices. Using simulations of single-shot images, we demonstrate that the fragmentation and correlations can be detected via the variance of the entropy of the images.

Our work highlights the importance of deploying modern computational and theoretical many-body approaches like the MCTDH-X to systems with artificial gauge fields as well as the necessity to consider not only the wavefunctions of ultracold atoms themselves, but also their detection.

Our results complement the recent findings, that the variances of observables are sensitive probes of correlations in the state of ultracold atomic systems $[9,38-43,96,98]$.

Straightforward continuations of this investigation include the deployment of the developed analysis and computational tools to other many-body systems. As examples of interest, we name here the variance of the image entropy in ultracold dipolar atoms as discussed in Refs. [44,48,49] and an exploration of the competition of long-ranged dipolar interactions and artificial magnetic fields for two-dimensional ultracold atoms. Another direction of physical interest is the dynamics of two-dimensional bosonic Josephson junctions [99] subject to gauge fields and the resulting tunneling of (phantom) vortices or the emergence of quantum turbulence [100] via entropy production [101], which, in turn, as we have shown above, could result from the presence of artificial gauge fields.

Author Contributions: A.U.J.L. performed computations, A.U.J.L. and S.D. implemented numerical algorithms, and A.U.J.L., S.D., and C.L. designed the study and wrote the manuscript. All authors have read and agreed to the published version of the manuscript.

Funding: This work and A.U.J.L. has been supported by the Austrian Science Foundation (FWF) under grant P-32033-N32. C.L. acknowledges funding by the FWF under grant M-2653 and by The Wiener Wissenschafts und Technologie Fonds (WWTF) project No. MA16-066. Computation time on the Hawk cluster at the HLRS Stuttgart is acknowledged. Support by the German Research Foundation (DFG) and the state of Baden-Württemberg via the bwHPC grants no INST 40/467-1 
FUGG (JUSTUS cluster), INST 39/963-1 FUGG (bwForCluster NEMO), and INST 37/935-1 FUGG (bwForCluster BinAC) is gratefully acknowledged.

Data Availability Statement: The MCTDH-X software to recompute all the results in this study is available at http:/ / ultracold.org (accessed on $\pi$-day, 14 March 2021). Results can be made available upon request; the total amount of data is several TB.

Acknowledgments: Insightful discussions with Saurabh Basu and Ofir E. Alon are gratefully acknowledged.

Conflicts of Interest: The authors declare no conflict of interest.

\section{Appendix A. The Equations of Motion of the Multiconfigurational Time-Dependent Hartree Method for Indistinguishable Particles}

We now discuss the equations of motion of the MCTDH-X. For simplicity, we present them using the gauge condition $\left\langle\dot{\phi}_{i} \mid \phi_{j}\right\rangle=0$; for other possible gauges, see for instance [22,102].

$$
\begin{aligned}
i \partial_{t}\left|\phi_{j}\right\rangle & =\hat{\mathbf{P}}\left[\hat{h}\left|\phi_{j}\right\rangle+\sum_{k, s, q, l=1}^{M}\{\underline{\rho}\}_{j k}^{-1} \rho_{k s l q} \hat{W}_{s l}(\mathbf{x} ; t)\left|\phi_{q}\right\rangle\right] ; \quad \hat{\mathbf{P}}=\mathbf{1}-\sum_{i}\left|\phi_{i}\right\rangle\left\langle\phi_{i}\right|,(\mathrm{A} 1) \\
i \partial_{t} C_{\vec{n}}(t) & =\sum_{\overrightarrow{n^{\prime}}}\left\langle\vec{n} ; t|\mathcal{H}| \overrightarrow{n^{\prime}} ; t\right\rangle C_{\vec{n}^{\prime}} .
\end{aligned}
$$

Here, we introduced the matrix elements of the reduced one-body and two-body density matrices,

$$
\begin{aligned}
\rho_{k q} & =\left\langle\Psi\left|\hat{b}_{k}^{+} \hat{b}_{q}\right| \Psi\right\rangle, \\
\rho_{k s l q} & =\left\langle\Psi\left|\hat{b}_{k}^{+} \hat{b}_{s}^{+} \hat{b}_{q} \hat{b}_{l}\right| \Psi\right\rangle,
\end{aligned}
$$

respectively. The $\{\underline{\rho}\}_{j k}^{-1}$ notation denotes the $j k$-th matrix element the inverse of a matrix filled with the entries defined in Equation (A3).

Moreover, the time-dependent local interaction potentials were used

$$
\hat{W}_{s l}(\mathbf{x} ; t)=\int \phi_{s}^{*}\left(\mathbf{x}^{\prime} ; t\right) \hat{W}\left(\mathbf{x}, \mathbf{x}^{\prime} ; t\right) \phi_{l}\left(\mathbf{x}^{\prime} ; t\right) d \mathbf{x}^{\prime} .
$$

In this work, we solve the Equations (A1) and(A2) with the MCTDH-X software [74] hosted at http:/ / ultracold.org (accessed on $\pi$-day, 14 March 2021).

\section{Appendix B. Investigation of Convergence}

Here, we discuss the convergence of our numerical simulations with respect to the number of variational parameters in the ansatz of the MCTDH-X wavefunction. For this purpose, we consider the same systems as investigated in the main text, but perform the solutions of the Schrödinger equation with MCTDH-X with a different number of orbitals $M$.

Figures $\mathrm{A} 1$ and $\mathrm{A} 2$ show the result for the first eigenvalue $\lambda_{1}$ of the $1 \mathrm{BDM}$ for $B=1$ and $B=5$, respectively, as a function of time for $M \leq 5$.

The time-evolution of $\lambda_{1}$ is not fully converged for $M \leq 5$. However, all shown timeevolutions with $M>1$ show a significant departure from the mean-field case, i.e., $\lambda_{1}<1$ for $M>1$. Importantly, the magnitude of the departure from the mean-field case increases drastically as the strength of the AMF is increased (cf. ranges of $\lambda_{1}$ in Figures A1 and A2). We note that the number of time-dependent configurations $\left|n_{1}, \ldots, n_{5} ; t\right\rangle$ for $N=100$ and $M=5$ is $4598126 \approx 4.6 \times 10^{6}$ and the total number of optimized and fully time-dependent parameters in these demanding computations is thus $4598126+5 \times 128^{2} \times 4=4680046 \approx$ $4.7 \times 10^{6}$. 
To verify this finding about $\lambda_{1}$ in a setting, where a larger number of orbitals can be included in our simulations, we repeated our convergence study in a system with $N=10$ particles. To keep the mean-field interaction $g_{0}(N-1)$ and predictions comparable, we investigated a stronger interaction of $g_{0}=0.5$ in this case.

Figures $\mathrm{A} 3$ and $\mathrm{A} 4$ show the $N=10$ results for the first eigenvalue $\lambda_{1}$ of the 1BDM for $B=1$ and $B=5$, respectively, as a function of time for $M \leq 13$.

A complete convergence of the eigenvalue $\lambda_{1}(t)$ is still not achieved; however, the discrepancies for different $M$ are very small, cf. the $M=12$ and $M=13$ cases in Figure A3 and the $M=11$ and $M=12$ cases in Figure A4. The tendency to an increased amount of fragmentation and correlations for an increased AMF field strength inferred in the $N=100$ case in Figures $A 1$ and $A 2$ is also underpinned by the results for $N=10: B=5$ yields a drastically smaller $\lambda_{1}(t)$ than $B=1$ (cf. ranges of $\lambda_{1}$ in Figures A3 and A4). At this stage, the discrepancies may well arise due to the formal problems with the true contact interaction potential $[75,76]$ that we have chosen to use for our present investigation. We note that the number of time-dependent configurations $\left|n_{1}, \ldots, n_{1} 3 ; t\right\rangle$ for $N=10$ and $M=13$ is $646646 \approx 6 \times 10^{5}$ and the total number of optimized and fully time-dependent parameters in these demanding computations is thus $646646+13 \times 128^{2} \times 4=859638 \approx$ $9 \times 10^{5}$.

In summary, our convergence study corroborates our main observations about the emergence of fragmentation and correlations in the main text and underpins the accuracy of our MCTDH-X computations.

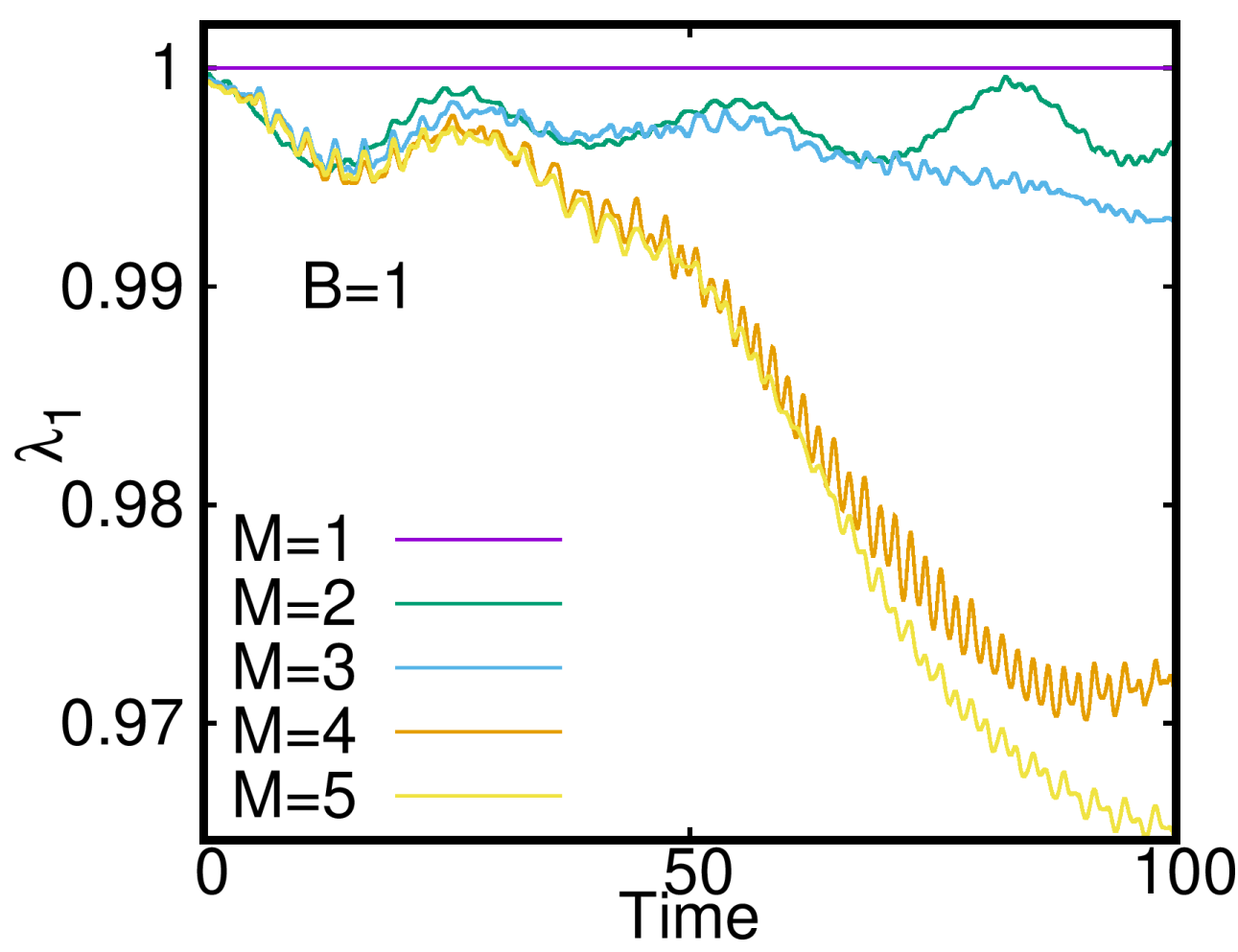

Figure A1. Convergence: $\lambda_{1}(t)$ for different $M$ with $N=100$ and $B=1$. See text for details. 


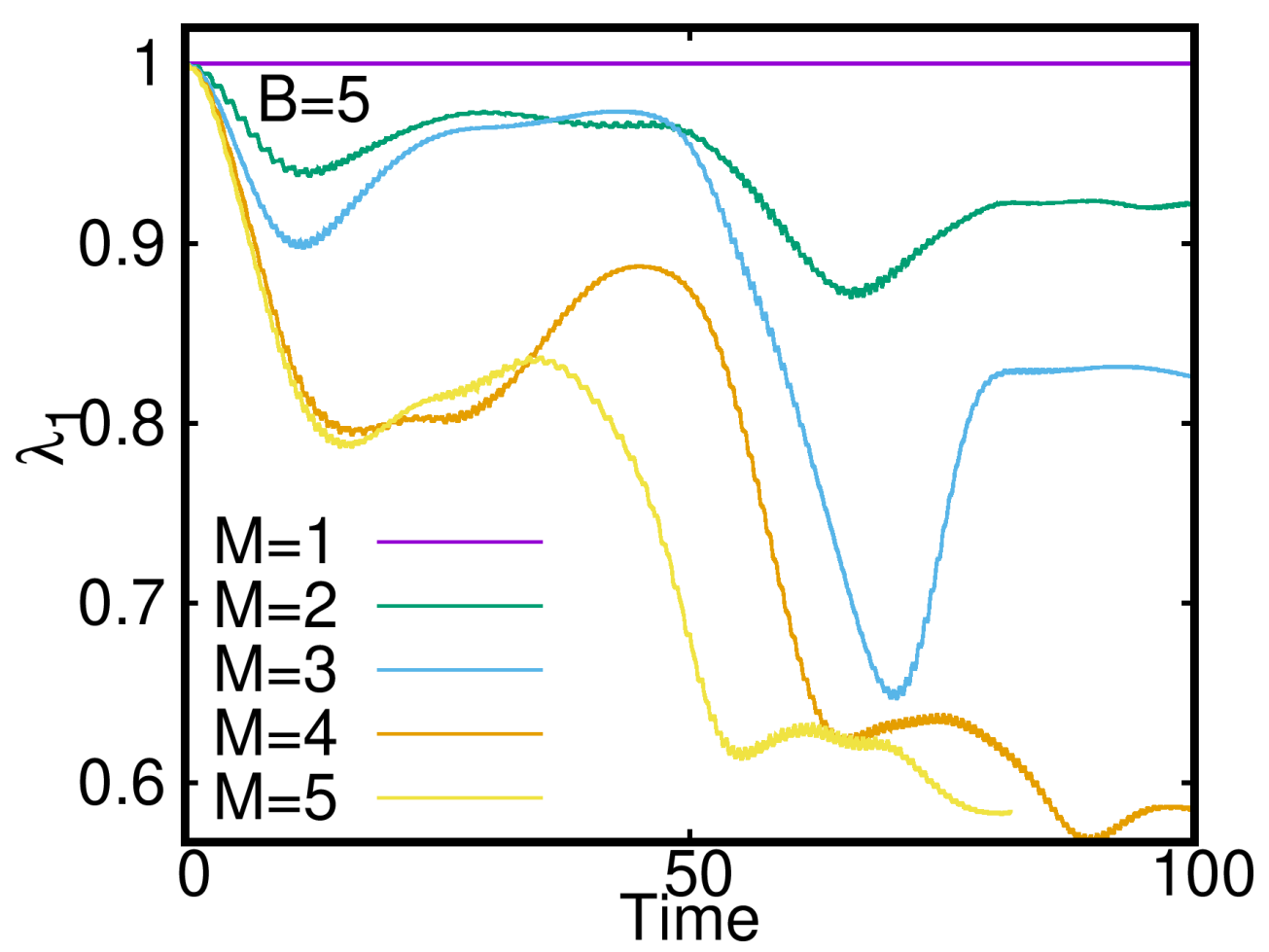

Figure A2. Convergence: $\lambda_{1}(t)$ for different $M$ with $N=100$ and $B=5$. The $M=5$ computation was continued only up to time $\sim 80$, due to the disproportionately large amount of computational resources required. See text for details.

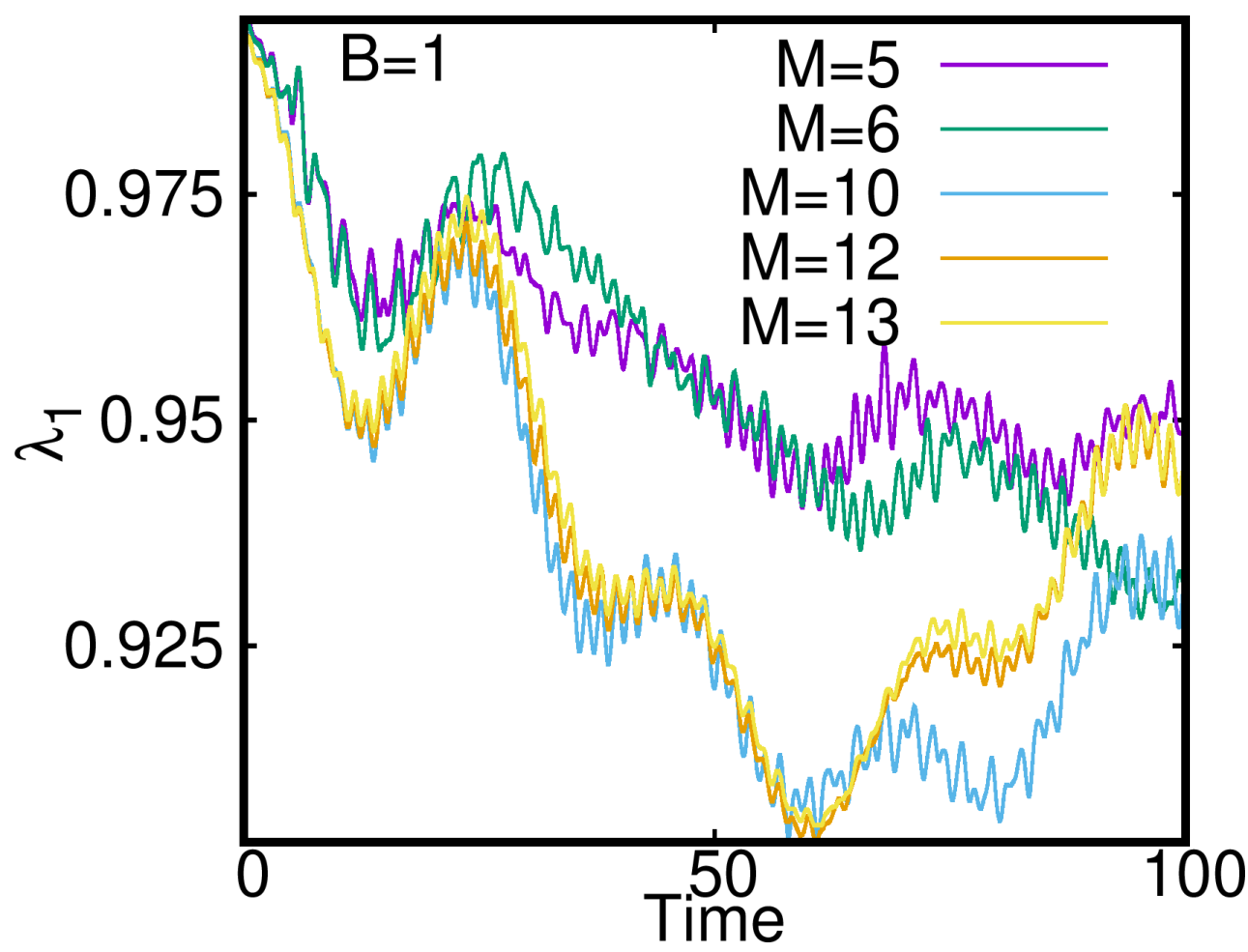

Figure A3. Convergence: $\lambda_{1}(t)$ for different $M$ with $N=10$ and $B=1$. See text for details. 


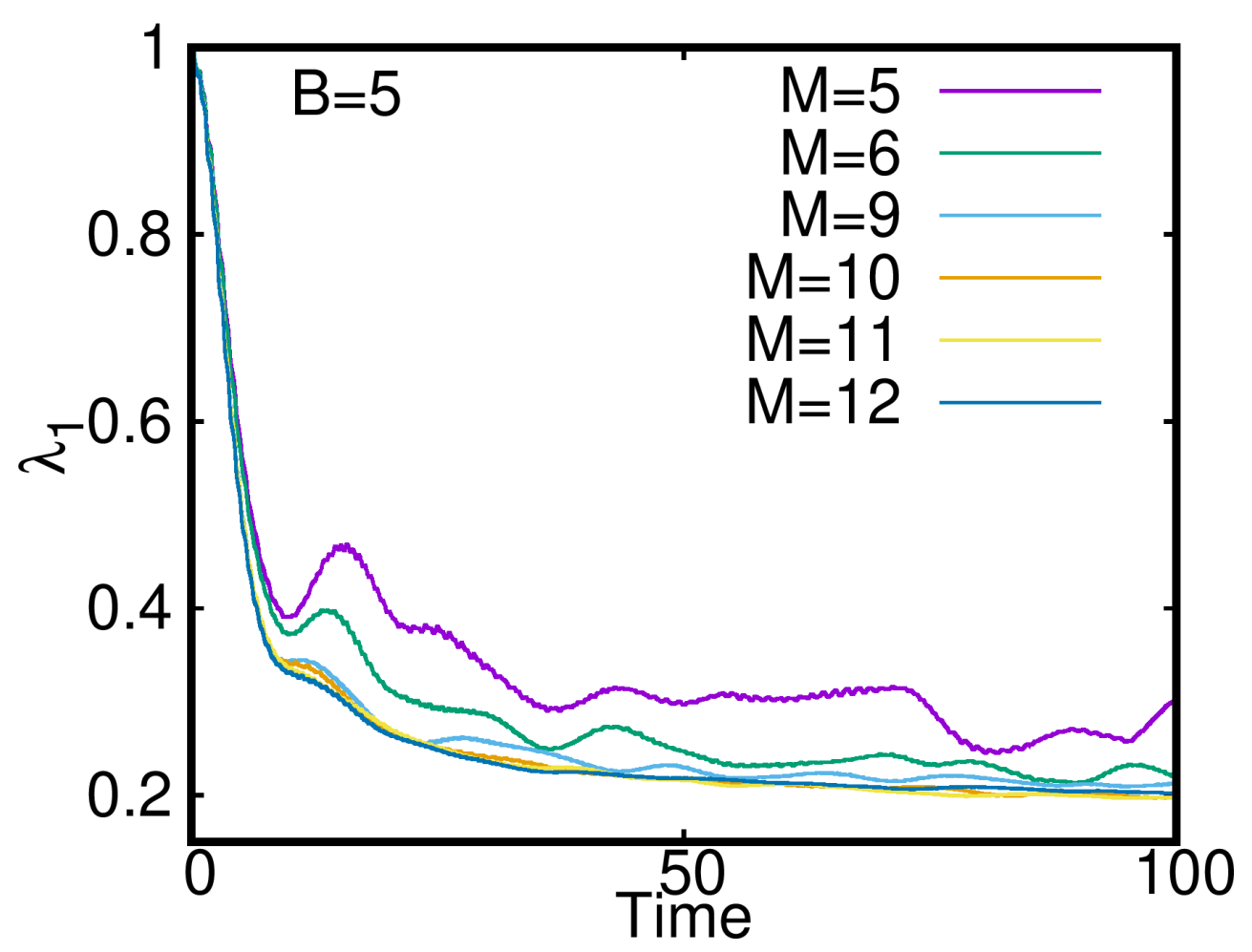

Figure A4. Convergence: $\lambda_{1}(t)$ for different $M$ with $N=10$ and $B=5$. See text for details.

\section{References}

1. Davis, K.B.; Mewes, M.O.; Andrews, M.R.; Van Druten, N.J.; Durfee, D.S.; Kurn, D.M.; Ketterle, W. Bose-Einstein condensation in a gas of sodium atoms. Phys. Rev. Lett. 1995, 75, 3969-3973. [CrossRef] [PubMed]

2. Anderson, M.H.; Ensher, J.R.; Matthews, M.R.; Wieman, C.E.; Cornell, E.A. Observation of bose-einstein condensation in a dilute atomic vapor. Collect. Pap. Carl Wieman 2008, 269, 453-456. [CrossRef]

3. Bradley, C.C.; Sackett, C.A.; Tollett, J.J.; Hulet, R.G. Evidence of Bose-Einstein condensation in an atomic gas with attractive interactions. Phys. Rev. Lett. 1995, 75, 1687-1690. [CrossRef] [PubMed]

4. Jaksch, D.; Bruder, C.; Cirac, J.I.; Gardiner, C.W.; Zoller, P. Cold bosonic atoms in optical lattices. Phys. Rev. Lett. 1998, 81, 3108-3111. [CrossRef]

5. Greiner, M.; Mandel, O.; Rom, T.; Altmeyer, A.; Widera, A.; Hänsch, T.W.; Bloch, I. Quantum phase transition from a superfluid to a Mott insulator in an ultracold gas of atoms. Phys. B Condens. Matter 2003, 329-333, 11-12. [CrossRef]

6. Krinner, S.; Stadler, D.; Husmann, D.; Brantut, J.P.; Esslinger, T. Observation of quantized conductance in neutral matter. Nature 2015, 517, 64-67. [CrossRef]

7. Corman, L.; Fabritius, P.; Häusler, S.; Mohan, J.; Dogra, L.H.; Husmann, D.; Lebrat, M.; Esslinger, T. Quantized conductance through a dissipative atomic point contact. Phys. Rev. A 2019, 100, 053605. [CrossRef]

8. Baumann, K.; Guerlin, C.; Brennecke, F.; Esslinger, T. Dicke quantum phase transition with a superfluid gas in an optical cavity. Nature 2010, 464, 1301. [CrossRef]

9. Lode, A.U.J.; Bruder, C. Fragmented Superradiance of a Bose-Einstein Condensate in an Optical Cavity. Phys. Rev. Lett. 2017, 118, 013603. [CrossRef]

10. Dalibard, J.; Gerbier, F.; Juzeliūnas, G.; Öhberg, P. Colloquium: Artificial gauge potentials for neutral atoms. Rev. Mod. Phys. 2011, 83, 1523. [CrossRef]

11. Aidelsburger, M. Artificial Gauge Fields with Ultracold Atoms in Optical Lattices; Springer International Publishing: Cham, Switzerland, 2016. [CrossRef]

12. Aidelsburger, M.; Atala, M.; Nascimbène, S.; Trotzky, S.; Chen, Y.A.; Bloch, I. Experimental Realization of Strong Effective Magnetic Fields in an Optical Lattice. Phys. Rev. Lett. 2011, 107, 255301. [CrossRef] [PubMed]

13. Lim, L.K.; Smith, C.M.; Hemmerich, A. Staggered-Vortex Superfluid of Ultracold Bosons in an Optical Lattice. Phys. Rev. Lett. 2008, 100, 130402. [CrossRef]

14. Spielman, I.B. Raman processes and effective gauge potentials. Phys. Rev. A 2009, 79, 063613. [CrossRef]

15. Lin, Y.J.; Compton, R.L.; Jiménez-García, K.; Porto, J.V.; Spielman, I.B. Synthetic magnetic fields for ultracold neutral atoms. Nature 2009, 462, 628-632. [CrossRef]

16. Lin, Y.J.; Compton, R.; Perry, A.; Phillips, W.; Porto, J.; Spielman, I. Bose-Einstein Condensate in a Uniform Light-Induced Vector Potential. Phys. Rev. Lett. 2009, 102, 130401. [CrossRef] 
17. Gross, E.P. Structure of a quantized vortex in boson systems. Nuovo Cim. Ser. 10 1961, 20, 454. [CrossRef]

18. Pitaevskii, L.P. Vortex Lines in an imperfect Bose gas. Sov. Phys. JETP 1961, 13, 451.

19. Streltsov, A.I.; Alon, O.E.; Cederbaum, L.S. Role of excited states in the splitting of a trapped interacting Bose-Einstein condensate by a time-dependent barrier. Phys. Rev. Lett. 2007, 99, 030402. [CrossRef] [PubMed]

20. Alon, O.E.; Streltsov, A.I.; Cederbaum, L.S. Multiconfigurational time-dependent Hartree method for bosons: Many-body dynamics of bosonic systems. Phys. Rev. A 2008, 77, 033613. [CrossRef]

21. Alon, O.E.; Streltsov, A.I.; Cederbaum, L.S. Unified view on multiconfigurational time propagation for systems consisting of identical particles. J. Chem. Phys. 2007, 127, 154103. [CrossRef]

22. Beck, M.H.; Jäckle, A.; Worth, G.A.; Meyer, H.D. The multiconfiguration time-dependent Hartree (MCTDH) method: A highly efficient algorithm for propagating wavepackets. Phys. Rep. 2000, 324, 1-105. [CrossRef]

23. Wang, H.; Thoss, M. Multilayer formulation of the multiconfiguration time-dependent Hartree theory. J. Chem. Phys. 2003, 119, 1289. [CrossRef]

24. Manthe, U. A multilayer multiconfigurational time-dependent Hartree approach for quantum dynamics on general potential energy surfaces. J. Chem. Phys. 2008, 128, 164116. [CrossRef]

25. Wang, H. Multilayer Multiconfiguration Time-Dependent Hartree Theory. J. Phys. Chem. A 2015, 119, 7951-7965. [CrossRef] [PubMed]

26. Manthe, U.; Weike, T. On the multi-layer multi-configurational time-dependent Hartree approach for bosons and fermions. J. Chem. Phys. 2017, 146, 064117. [CrossRef] [PubMed]

27. Manthe, U. Wavepacket dynamics and the multi-configurational time-dependent Hartree approach. J. Phys. Condens. Matter 2017, 29, 253001. [CrossRef]

28. Zanghellini, J.; Kitzler, M.; Fabian, C.; Brabec, T.; Scrinzi, A. An MCTDHF Approach to Multielectron Dynamics in Laser Fields. Laser Phys. 2003, 13, 1064.

29. Cao, L.; Krönke, S.; Vendrell, O.; Schmelcher, P. The multi-layer multi-configuration time-dependent Hartree method for bosons: Theory, implementation, and applications. J. Chem. Phys. 2013, 139, 134103. [CrossRef]

30. Cao, L.; Bolsinger, V.; Mistakidis, S.I.; Koutentakis, G.M.; Krönke, S.; Schurer, J.M.; Schmelcher, P. A unified ab initio approach to the correlated quantum dynamics of ultracold fermionic and bosonic mixtures. J. Chem. Phys. 2017, 147, 044106. [CrossRef]

31. Haxton, D.J.; McCurdy, C.W. Two methods for restricted configuration spaces within the multiconfiguration time-dependent Hartree-Fock method. Phys. Rev. A 2015, 91, 012509. [CrossRef]

32. Alon, O.E.; Streltsov, A.I.; Cederbaum, L.S. Multiconfigurational Time-Dependent Hartree Method for Mixtures Consisting of Two Types of Identical Particles; American Physical Society: College Park, MD, USA, 2007; Volume 76, p. 062501. [CrossRef]

33. Alon, O.E.; Streltsov, A.I.; Sakmann, K.; Lode, A.U.J.; Grond, J.; Cederbaum, L.S. Recursive formulation of the multiconfigurational time-dependent Hartree method for fermions, bosons and mixtures thereof in terms of one-body density operators. Chem. Phys. 2012, 401, 2-14. [CrossRef]

34. Miyagi, H.; Madsen, L.B. Time-dependent restricted-active-space self-consistent-field theory for laser-driven many-electron dynamics. Phys. Rev. A 2013, 87, 062511. [CrossRef]

35. Miyagi, H.; Madsen, L.B. Time-Dependent Restricted-Active-Space Self-Consistent-Field Theory With Space Partition; American Physical Society: College Park, MD, USA, 2017; Volume 95, p. 023415. [CrossRef]

36. Lévêque, C.; Madsen, L.B. Time-dependent restricted-active-space self-consistent-field theory for bosonic many-body systems. New J. Phys. 2017, 19, 043007. [CrossRef]

37. Lévêque, C.; Madsen, L.B. Multispecies time-dependent restricted-active-space self-consistent-field theory for ultracold atomic and molecular gases. J. Phys. B At. Mol. Opt. Phys. 2018, 51, 155302. [CrossRef]

38. Lode, A.U.J.; Lévêque, C.; Madsen, L.B.; Streltsov, A.I.; Alon, O.E. Colloquium : Multiconfigurational time-dependent Hartree approaches for indistinguishable particles. Rev. Mod. Phys. 2020, 92, 011001. [CrossRef]

39. Klaiman, S.; Alon, O.E. Variance as a sensitive probe of correlations. Phys. Rev. A 2015, 91, 063613. [CrossRef]

40. Klaiman, S.; Streltsov, A.I.; Alon, O.E. Uncertainty Product of an Out-of-Equilibrium Many-Particle System; American Physical Society: College Park, MD, USA, 2016; Volume 93, p. 023605. [CrossRef]

41. Klaiman, S.; Beinke, R.; Cederbaum, L.S.; Streltsov, A.I.; Alon, O.E. Variance of an anisotropic Bose-Einstein condensate. Chem. Phys. 2018, 509, 45-54. [CrossRef]

42. Alon, O.E. Condensates in annuli: Dimensionality of the variance. Mol. Phys. 2019, 117, 2108-2120. [CrossRef]

43. Alon, O.E. Analysis of a trapped Bose-Einstein condensate in terms of position, momentum, and angular-momentum variance. Symmetry 2019, 11, 1344. [CrossRef]

44. Chatterjee, B.; Lode, A.U.J. Order parameter and detection for a finite ensemble of crystallized one-dimensional dipolar bosons in optical lattices. Phys. Rev. A 2018, 98, 053624. [CrossRef]

45. Mistakidis, S.I.; Cao, L.; Schmelcher, P. Interaction quench induced multimode dynamics of finite atomic ensembles. J. Phys. B At. Mol. Opt. Phys. 2014, 47, 225303. [CrossRef]

46. Mistakidis, S.I.; Cao, L.; Schmelcher, P. Negative-quench-induced excitation dynamics for ultracold bosons in one-dimensional lattices. Phys. Rev. A 2015, 91, 033611. [CrossRef]

47. Mistakidis, S.I.; Schmelcher, P. Mode coupling of interaction quenched ultracold few-boson ensembles in periodically driven lattices. Phys. Rev. A 2017, 95, 013625. [CrossRef] 
48. Chatterjee, B.; Tsatsos, M.C.; Lode, A.U.J. Correlations of strongly interacting one-dimensional ultracold dipolar few-boson systems in optical lattices. New J. Phys. 2019, 21, 033030. [CrossRef]

49. Chatterjee, B.; Lévêque, C.; Schmiedmayer, J.; Lode, A.U.J. Detecting One-Dimensional Dipolar Bosonic Crystal Orders via Full Distribution Functions. Phys. Rev. Lett. 2020, 125, 093602. [CrossRef] [PubMed]

50. Lin, R.; Molignini, P.; Papariello, L.; Tsatsos, M.C.; Lévêque, C.; Weiner, S.E.; Fasshauer, E.; Chitra, R.; Lode, A.U.J. MCTDH-X : The multiconfigurational time-dependent Hartree method for indistinguishable particles software. Quantum Sci. Technol. 2020, 5, 024004. [CrossRef]

51. Lode, A.U.J.; Diorico, F.S.; Wu, R.; Molignini, P.; Papariello, L.; Lin, R.; Lévêque, C.; Exl, L.; Tsatsos, M.C.; Chitra, R.; et al. Many-body physics in two-component Bose-Einstein condensates in a cavity: Fragmented superradiance and polarization. New $\mathrm{J}$ Phys. 2018, 20, 055006. [CrossRef]

52. Lin, R.; Papariello, L.; Molignini, P.; Chitra, R.; Lode, A.U.J. Superfluid-Mott-insulator transition of ultracold superradiant bosons in a cavity. Phys. Rev. A 2019, 100, 013611. [CrossRef]

53. Lin, R.; Molignini, P.; Lode, A.U.J.; Chitra, R. Pathway to chaos through hierarchical superfluidity in blue-detuned cavity-BEC systems. Phys. Rev. A 2020, 101, 061602. [CrossRef]

54. Lode, A.U.J.; Streltsov, A.I.; Sakmann, K.; Alon, O.E.; Cederbaum, L.S. How an interacting many-body system tunnels through a potential barrier to open space. Proc. Natl. Acad. Sci. USA 2012, 109, 13521. [CrossRef]

55. Lode, A.U.J.; Klaiman, S.; Alon, O.E.; Streltsov, A.I.; Cederbaum, L.S. Controlling the velocities and the number of emitted particles in the tunneling to open space dynamics. Phys. Rev. A 2014, 89, 053620. [CrossRef]

56. Koutentakis, G.M.; Mistakidis, S.I.; Schmelcher, P. Quench-induced resonant tunneling mechanisms of bosons in an optical lattice with harmonic confinement. Phys. Rev. A 2017, 95, 013617. [CrossRef]

57. Klaiman, S.; Cederbaum, L.S. Overlap of exact and Gross-Pitaevskii wave functions in Bose-Einstein condensates of dilute gases. Phys. Rev. A 2016, 94, 063648. [CrossRef]

58. Nguyen, J.H.V.; Tsatsos, M.C.; Luo, D.; Lode, A.U.J.; Telles, G.D.; Bagnato, V.S.; Hulet, R.G. Parametric Excitation of a Bose-Einstein Condensate: From Faraday Waves to Granulation. Phys. Rev. X 2019, 9, 011052. [CrossRef]

59. Bera, S.; Chakrabarti, B.; Gammal, A.; Tsatsos, M.C.; Lekala, M.L.; Chatterjee, B.; Lévêque, C.; Lode, A.U.J. Sorting Fermionization from Crystallization in Many-Boson Wavefunctions. Sci. Rep. 2019, 9, 17873. [CrossRef]

60. Nozières, P.; St. James, D. Particle Vs. Pair Condensation in Attractive Bose Liquids. J. Phys. Paris 1982, 43, 1133-1148. [CrossRef]

61. Spekkens, R.W.; Sipe, J.E. Spatial fragmentation of a Bose-Einstein condensate in a double-well potential. Phys. Rev. A 1999, 59, 3868-3877. [CrossRef]

62. Mueller, E.J.; Ho, T.L.; Ueda, M.; Baym, G. Fragmentation of Bose-Einstein condensates. Phys. Rev. A 2006, 74, 033612. [CrossRef]

63. Streltsov, A.I.; Alon, O.E.; Cederbaum, L.S. Efficient generation and properties of mesoscopic quantum superposition states in an attractive Bose-Einstein condensate threaded by a potential barrier. J. Phys. B At. Mol. Opt. Phys. 2009, 42, 091004. [CrossRef]

64. Streltsov, A.I.; Alon, O.E.; Cederbaum, L.S. Scattering of an attractive Bose-Einstein condensate from a barrier: Formation of quantum superposition states. Phys. Rev. A 2009, 80, 043616. [CrossRef]

65. Streltsov, A.I.; Alon, O.E.; Cederbaum, L.S. Formation and dynamics of many-boson fragmented states in one-dimensional attractive ultracold gases. Phys. Rev. Lett. 2008, 100, 130401. [CrossRef]

66. Sakmann, K. Many-Body Schrödinger Dynamics of Bose-Einstein Condensates; Springer: Berlin/Heidelberg, Germany, 2011. [CrossRef]

67. Streltsov, A.I.; Alon, O.E.; Cederbaum, L.S. Swift loss of coherence of soliton trains in attractive bose-einstein condensates. Phys. Rev. Lett. 2011, 106, 240401. [CrossRef] [PubMed]

68. Beinke, R.; Klaiman, S.; Cederbaum, L.S.; Streltsov, A.I.; Alon, O.E. Many-body tunneling dynamics of Bose-Einstein condensates and vortex states in two spatial dimensions. Phys. Rev. A 2015, 92, 043627. [CrossRef]

69. Weiner, S.E.; Tsatsos, M.C.; Cederbaum, L.S.; Lode, A.U.J. Phantom vortices: Hidden angular momentum in ultracold dilute Bose-Einstein condensates. Sci. Rep. 2017, 7, 40122. [CrossRef]

70. Dutta, S.; Tsatsos, M.C.; Basu, S.; Lode, A.U.J. Management of the correlations of Ultracold Bosons in triple wells. New J. Phys. 2019, 21, 053044. [CrossRef]

71. Fasshauer, E.; Lode, A.U.J. Multiconfigurational time-dependent Hartree method for fermions: Implementation, exactness, and few-fermion tunneling to open space. Phys. Rev. A 2016, 93, 033635. [CrossRef]

72. Lode, A.U.J. Multiconfigurational time-dependent Hartree method for bosons with internal degrees of freedom: Theory and composite fragmentation of multicomponent Bose-Einstein condensates. Phys. Rev. A 2016, 93, 063601. [CrossRef]

73. Lode, A.U.J.; Bruder, C. Dynamics of Hubbard Hamiltonians with the multiconfigurational time-dependent Hartree method for indistinguishable particles. Phys. Rev. A 2016, 94, 013616. [CrossRef]

74. Lode, A.U.J.; Tsatsos, M.C.; Fasshauer, E.; Lin, R.; Papariello, L.; Molignini, P.; Weiner, S.E.; Lévêque, C. MCTDH-X: The Multiconfigurational Time-Dependent Hartree For Indistinguishable Particles Software. http://ultracold.org (accessed on 14 March 2021).

75. Doganov, R.A.; Klaiman, S.; Alon, O.E.; Streltsov, A.I.; Cederbaum, L.S. Two trapped particles interacting by a finite-range two-body potential in two spatial dimensions. Phys. Rev. A 2013,87, 033631. [CrossRef]

76. Friedman, C.N. Perturbations of the Schroedinger equation by potentials with small support. J. Funct. Anal. 1972, 10, 346-360. [CrossRef] 
77. Dirac, P.A.M. Note on Exchange Phenomena in the Thomas Atom. Math. Proc. Camb. Philos. Soc. 1930, 26, 376. [CrossRef]

78. Mott, N.F.; Frenkel, J. Wave Mechanics: Advanced General Theory; Clarendon Press: Oxford, UK, 1934; Volume 18, p. 208. [CrossRef]

79. McLachlan, A.D. A variational solution of the time-dependent Schrodinger equation. Mol. Phys. 1964, 8, 39-44. [CrossRef]

80. Kramer, P.; Saraceno, M. Geometry of the Time-Dependent Variational Principle in Quantum Mechanics; Springer: Berlin/Heidelberg, Germany, 2007. [CrossRef]

81. Kvaal, S. Variational formulations of the coupled-cluster method in quantum chemistry. Mol. Phys. 2013, 111, 1100-1108. [CrossRef]

82. Penrose, O.; Onsager, L. Bose-einstein condensation and liquid helium. Phys. Rev. 1956, 104, 576-584. [CrossRef]

83. Sakmann, K.; Streltsov, A.I.; Alon, O.E.; Cederbaum, L.S. Reduced density matrices and coherence of trapped interacting bosons. Phys. Rev. A 2008, 78, 023615. [CrossRef]

84. Wells, T.; Lode, A.U.J.; Bagnato, V.S.; Tsatsos, M.C. Vortex Reconnections in Anisotropic Trapped Three-Dimensional Bose-Einstein Condensates. J. Low Temp. Phys. 2015, 180, 133-143. [CrossRef]

85. Sakmann, K.; Kasevich, M. Single-shot simulations of dynamic quantum many-body systems. Nat. Phys. 2016, 12, 451. [CrossRef]

86. Klaiman, S.; Alon, O.E. Spatially-partitioned many-body vortices. J. Phys. Conf. Ser. 2016, 691, 012015. [CrossRef]

87. Tsatsos, M.C.; Streltsov, A.I.; Alon, O.E.; Cederbaum, L.S. Fragmented many-body states of definite angular momentum and stability of attractive three-dimensional condensates. Phys. Rev. A 2010, 82, 33613. [CrossRef]

88. Tsatsos, M.C.; Lode, A.U.J. Resonances and Dynamical Fragmentation in a Stirred Bose-Einstein Condensate. J. Low Temp. Phys. 2015, 181, 171-181. [CrossRef]

89. Gajda, M.; Mostowski, J.; Sowiński, T.; Załuska-Kotur, M. Single-shot imaging of trapped Fermi gas. Europhys. Lett. 2016, 115, 20012. [CrossRef]

90. Pyzh, M.; Krönke, S.; Weitenberg, C.; Schmelcher, P. Quantum point spread function for imaging trapped few-body systems with a quantum gas microscope. New J. Phys. 2019, 21, 053013. [CrossRef]

91. Roy, R.; Gammal, A.; Tsatsos, M.C.; Chatterjee, B.; Chakrabarti, B.; Lode, A.U.J. Phases, many-body entropy measures, and coherence of interacting bosons in optical lattices. Phys. Rev. A 2018, 97, 043625. [CrossRef]

92. Tsubota, M.; Kasamatsu, K.; Ueda, M. Vortex lattice formation in a rotating Bose-Einstein condensate. Phys. Rev. A 2002, 65, 023603. [CrossRef]

93. Dagnino, D.; Barberán, N.; Lewenstein, M. Vortex nucleation in a mesoscopic Bose superfluid and breaking of the parity symmetry. Phys. Rev. A 2009, 80, 53611. [CrossRef]

94. Alon, O.E.; Bagnato, V.S.; Beinke, R.; Brouzos, I.; Calarco, T.; Caneva, T.; Cederbaum, L.S.; Kasevich, M.A.; Klaiman, S.; Lode, A.U.J.; et al. MCTDHB physics and technologies: Excitations and vorticity, single-shot detection, measurement of fragmentation, and optimal control in correlated ultra-cold bosonic many-body Systems. In High Performance Computing in Science and Engineering '15; Springer: Cham, Switzerland, 2016; pp. 23-49. [CrossRef]

95. Cremon, J.C.; Jackson, A.D.; Karabulut, E.Ö.; Kavoulakis, G.M.; Mottelson, B.R.; Reimann, S.M. Rotating Bose-Einstein condensates: Closing the gap between exact and mean-field solutions. Phys. Rev. A 2015, 91, 033623. [CrossRef]

96. Alon, O.E.; Beinke, R.; Bruder, C.; Cederbaum, L.S.; Klaiman, S.; Lode, A.U.J.; Sakmann, K.; Theisen, M.; Tsatsos, M.C.; Weiner, S.E.; et al. Many-Body Effects in Fragmented, Depleted, and Condensed Bosonic Systems in Traps and Optical Cavities by MCTDHB and MCTDH-X; Springer: Berlin/Heidelberg, Germany, 2018; pp. 93-115. [CrossRef]

97. Eriksson, G.; Bengtsson, J.; Kavoulakis, G.M.; Reimann, S.M. Two-state model for vortex nucleation in a rotating Bose-Einstein condensate. Phys. Rev. A 2019, 100, 063638. [CrossRef]

98. Alon, O.E.; Cederbaum, L.S. Attractive Bose-Einstein condensates in anharmonic traps: Accurate numerical treatment and the intriguing physics of the variance. Chem. Phys. 2018, 515, 287-298. [CrossRef]

99. Bhowmik, A.; Haldar, S.K.; Alon, O.E. Impact of the transverse direction on the many-body tunneling dynamics in a twodimensional bosonic Josephson junction. Sci. Rep. 2020, 10, 21476. [CrossRef]

100. Tsatsos, M.C.; Tavares, P.E.; Cidrim, A.; Fritsch, A.R.; Caracanhas, M.A.; dos Santos, F.E.A.; Barenghi, C.F.; Bagnato, V.S. Quantum turbulence in trapped atomic Bose-Einstein condensates. Phys. Rep. 2016, 622, 1-52. [CrossRef]

101. Madeira, L.; García-Orozco, A.D.; dos Santos, F.E.A.; Bagnato, V.S. Entropy of a Turbulent Bose-Einstein Condensate. Entropy 2020, 22, 956. [CrossRef] [PubMed]

102. Caillat, J.; Zanghellini, J.; Kitzler, M.; Koch, O.; Kreuzer, W.; Scrinzi, A. Correlated multielectron systems in strong laser fields: A multiconfiguration time-dependent Hartree-Fock approach. Phys. Rev. A 2005, 71, 012712. [CrossRef] 